\title{
EL CONSUMO DE TOMATES PREVIENE EL DESARROLLO DE ENFERMEDADES CARDIOVASCULARES Y CÁNCER: ANTECEDENTES EPIDEMIOLÓGICOS Y MECANISMOS DE ACCIÓN
}

\author{
TOMATO CONSUMPTION PREVENTS THE DEVELOPMENT OF \\ CARDIOVASCULAR EVENTS AND CANCER: EPIDEMIOLOGIC ANTECEDENTS \\ AND ACTION MECHANISMS
}

Iván Palomo ${ }^{1}$; Rodrigo Moore-Carrasco ${ }^{1}$; Gilda Carrasco ${ }^{2}$; Pablo Villalobos ${ }^{3}$; Luis Guzmán ${ }^{1}$

\begin{abstract}
RESUMEN
Las enfermedades no transmisibles (ENT), especialmente las enfermedades cardiovasculares (ECV) y el cáncer, representan un importante problema de salud pública. Una de las estrategias para revertir esta situación es incrementar el consumo de frutas y hortalizas. Por lo anterior, esta revisión muestra los hallazgos epidemiológicos y mecanismos por los cuales la ingesta de tomate podría prevenir el desarrollo de ECV y cáncer. El tomate, rico en licopeno, es la hortaliza de mayor consumo a nivel mundial y la que más se cultiva en Chile. Desde la década de los noventa, varios estudios epidemiológicos han mostrado que el consumo de tomate puede prevenir el desarrollo de eventos cardiovasculares (infarto agudo de miocardio y enfermedad cerebrovascular) y ciertos tipos de cánceres. Respecto a los mecanismos protectores de ECV se ha observado que el tomate presenta actividades antiplaquetaria, protectora del endotelio, antioxidante y antiaterogénica. Por su parte, entre los mecanismos por los cuales puede prevenir el cáncer, se han descrito las siguientes actividades: antioxidante, activación de apoptosis, disminución de la proliferación celular y disminución de la angiogénesis y metástasis. A futuro será necesario avanzar en el conocimiento de los mecanismos moleculares involucrados (células, receptores y vías de señalización). Por otra parte, teniendo en consideración el aporte del tomate y sus productos en la prevención de las ENT, junto con incrementar las campañas sobre consumo interno y exportación, será fundamental idear nuevos alimentos funcionales y nutracéuticos.
\end{abstract}

Palabras clave: Tomate, enfermedades cardiovasculares, cáncer, antioxidantes.

\begin{abstract}
Non-transmissible diseases (NTDs), especially cardiovascular disease (CVD) and cancer, are a major public health problem. One strategy to reverse this situation is to increase consumption of fruits and vegetables. For these reasons, this review shows the epidemiological findings and mechanisms by which tomato intake may prevent the development of CVD and cancer. The tomato rich in lycopene is the most consumed vegetable in the world and the main crop in Chile. Since the nineties, several epidemiological studies have shown that tomato consumption may prevent the development of cardiovascular events (myocardial infarction and cerebrovascular disease) and certain types of cancers. Regarding the protective mechanisms of CVD, it has been observed that the tomato has antiplatelet activity, endothelial protective, antioxidant and antiatherogenic. Meanwhile, among the mechanisms by which it can prevent cancer has described the following activities: antioxidant, activation of apoptosis, decreased cell proliferation and angiogenesis and diminution of metastasis. In the future it is necessary to advance the knowledge of the molecular mechanisms involved (cells, receptors and signaling pathways). Also, taking into consideration the contribution of the tomato and its products in the prevention of NTD, along with the outstanding campaigns to increase domestic consumption and export, it will be essential to devise new functional and nutraceutical food.
\end{abstract}

Key words: Tomato, cardiovascular diseases, cancer, antioxidants.

1 Programa de Investigación en Factores de Riesgo de Enfermedades Cardiovasculares (PIFRECV), Departamento de Bioquímica Clínica e Inmunohematología, Facultad de Ciencias de la Salud, Universidad de Talca.

2 Departamento de Horticultura, Facultad de Ciencias Agrarias, Universidad de Talca.

3 Departamento de Economía Agraria, Facultad de Ciencias Agrarias, Universidad de Talca.

Fecha de Recepción: 30 Octubre, 2009

Fecha de Aceptación: 14 Diciembre, 2009 


\section{INTRODUCCIÓN}

Las enfermedades no transmisibles (ENT), entre otras las enfermedades cardiovasculares (ECV) y el cáncer, representan un problema de salud pública a nivel mundial (1), situación de la que Chile no está ajeno (2-4). Varios estudios han mostrado la alta prevalencia de factores de riesgo de ENT en la población adulta chilena $(5,6)$

El informe sobre "Dieta, nutrición y prevención de enfermedades crónicas" publicado por la Organización Mundial de la Salud (OMS) en el año 2003, reconoció que la evidencia científica asociada a la disminución del riesgo de ECV en las personas que consumen al menos $400 \mathrm{~g}$ de frutas y hortalizas al día era convincente y un elemento probable en la disminución del riesgo de algunos tipos de cánceres (7). Por su parte, el Fondo Internacional para la Investigación del Cáncer (WCRF) y el Instituto Americano de Investigación del Cáncer (AICR), en su informe "Alimentación, nutrición, actividad física y prevención del cáncer: una perspectiva mundial", confirman la evidencia científica entre la asociación de distintos tipos de cánceres y la alimentación, la actividad física y el peso de las personas (8). En la actualidad, los beneficios nutricionales del consumo de frutas y hortalizas, además de su reconocido aporte en vitaminas, minerales y fibra dietética, se asocian a su contenido en fitoquímicos con efecto antioxidante o con acciones específicas sobre algunas enzimas, que a su vez llevan a cabo importantes funciones que contribuyen a la prevención de diversas ENT. En Chile, el consumo de frutas y hortalizas se estima en $160 \mathrm{~g} /$ persona/día, cifra muy inferior a los 400 $\mathrm{g} /$ persona/día recomendados por la OMS (9).

Como una forma de contribuir a los programas de promoción del consumo de frutas y hortalizas, esta revisión muestra las propiedades específicas del tomate asociadas a la prevención de ENT, ya que este fruto es la hortaliza que más se consume en el mundo (10).

\section{ANTECEDENTES DE TOMATE}

El tomate (Solanum lycopersicon Mill.) es una dicotiledónea perteneciente a la familia de las Solanáceas. Es una planta perenne cultivada como anual, se desarrolla ya sea rastrera, semierecta o erecta (11). Es la principal hortaliza de fruto en el mundo y de alto consumo como producto en fresco o procesado, ya sea en pasta, jugo, deshidratado, entre otros (10). Los principales productores de tomate en el mundo son China, Estados Unidos, Turquía, Italia e India. Chile se encuentra en el lugar 13 a nivel mundial considerando su producción para proceso (12). En Chile se cultiva en todo el país, mayoritariamente en suelo al aire libre o bajo invernadero, siendo la producción para consumo fresco, mayoritariamente interno, cercana a las 300 mil toneladas (13).

\section{LICOPENO Y OTROS ANTIOXIDANTES}

El tomate maduro, además de agua, posee carbohidratos, potasio, fósforo, magnesio, vitaminas B1, B2, B5 y C (14); también presenta carotenoides como el licopeno, el que junto a la vitamina $\mathrm{C}$ son antioxidantes $(15,16)$. En el tomate el principal carotenoide es el licopeno (83\%), constituyendo la base molecular para la síntesis de los restantes carotenoides. Es de estructura sencilla, con una cadena alifática formada por cuarenta átomos de carbono y un gran número de dobles enlaces conjugados (17) (Figura 1). La absorción del licopeno, por ser liposoluble, es mayor cuando se consume con aceite (18). En el organismo humano se le encuentra en el plasma $(30 \mu \mathrm{g} / \mathrm{dl})$ y en tejidos $(19,20)$.

El licopeno se acumula en los frutos de tomate desde su estado inmaduro con una tasa de incremento baja, aumentando significativamente ésta con la maduración del mismo. El contenido de licopeno<smiles>CC(C)=CC=CC(C)=CC=CC(C)=CC=CC=C(C)C=CC=C(C)C=CC=C(C)C</smiles>

Figura 1. Estructura del licopeno. 
puede presentar diferencias según la variedad y condiciones del cultivo y factores de postcosecha (21-23). Para híbridos de tomate destinados a proceso se ha observado un efecto varietal, el cual depende de la zona y fecha de cultivo. Así, en estudios realizados en la zona centro-sur de Chile el contenido promedio de licopeno obtenido en las temporadas 2002/03, 2003/04 y 2004/05 en la región del Maule, fue de 14,$10 ; 10,61$ y $14,63 \mathrm{mg} / 100 \mathrm{~g}$ (peso fresco) (22). Para frutos de consumo en fresco (tipo racimo, larga vida o "talquino") se desconocen valores promedio; sin embargo, la literatura internacional indica que un tomate de consumo en fresco en promedio posee un contenido de licopeno de aproximadamente $4 \mathrm{mg} / 100 \mathrm{~g}$ (peso fresco).

\section{TOMATE Y PREVENCIÓN DE ENFERMEDADES CARDIOVASCULARES (ECV)}

\section{EPIDEMIOLOGÍA}

Varios trabajos epidemiológicos han reportado que el tomate previene el desarrollo de eventos cardiovasculares (24-26). Entre otros, en 1994 se investigaron las razones para el bajo promedio de muertes por ECV en Nápoles, Italia, comparado con Bristol, Inglaterra, en el cual después de todos los controles los autores concluyeron que el consumo de tomate y aceite de oliva en Nápoles podría explicar las diferencias (27). A su vez, el European Study of Antioxidants, Myocardial Infarction, and Cancer of the Breast (EURAMIC) fue usado para examinar la relación entre la concentración de licopeno e infarto agudo de miocardio (28).

A continuación se revisan los principales mecanismos descritos por los cuales el licopeno y otros compuestos antioxidantes del tomate presentan dicho efecto protector.

\section{MECANISMOS PROTECTORES DE ECV}

Actividad antiplaquetaria. Al complejo proceso de hemostasia, clásicamente se le separa en hemostasia primaria, hemostasia secundaria (coagulación) y fibrinólisis. La hemostasia primaria incluye la vasoconstricción y la relación plaquetas-endotelio. Desde el punto de vista funcional, las plaquetas desarrollan fenómenos de adhesión, secreción y agregación. Las plaquetas se adhieren a la
Tabla 1

Mecanismos por los cuales el tomate previene las enfermedades cardiovasculares (ECV) y el cáncer.

\begin{tabular}{|c|}
\hline Prevención de ECV \\
\hline Antiagregación plaquetaria \\
Vía receptor de ADP \\
Vía receptor de colágeno \\
Protección de endotelio \\
\hline Disminución del LDL-c \\
\hline Inhibición de la HMG CoA reductasa \\
\hline Antioxidadación de la LDL \\
\hline Estimulación de la actividad del receptor de LDL-c. \\
\hline
\end{tabular}

Prevención de cáncer

Efecto antioxidante

Activación de apoptosis

Inhibición de la proliferación celular

Invasión celular, angiogénesis y metástasis

LDL, Low Density Lipoprotein; c, cholesterol, HMG CoA 3-Hidroxi-3-metilglutaril coenzima-A

matriz subendotelial a través de uniones entre sus glicoproteínas específicas de membrana (GPIa-IIa, GPIV, GPVI y GPIb-IX-V) y proteínas adhesivas del subendotelio, especialmente colágeno y factor von Willebrand (FVW) $(29,30)$.

Las plaquetas activadas favorecen la hemostasia secundaria (actividad procoagulante) al exponer fosfolípidos aniónicos (29) y expresar factor tisular (FT) (31). Desde el punto de vista fisiopatológico, se sabe que las plaquetas participan tanto en la aterogénesis (32) como en la trombosis arterial propiamente tal $(33,34)$. En ese sentido, la actividad antiagregante plaquetaria del tomate puede tener un efecto protector de ECV.

Se ha observado que la ingesta de tomate tiene actividad antiagregante plaquetaria in vitro e in vivo al inhibir la agregación inducida por ADP y colágeno, y se ha sugerido que las moléculas licopeno, adenosina y probablemente otras podrían ser responsables de dicho efecto (35-37).

Por otra parte, el diferente potencial antitrombótico observado en distintas variedades de tomates podría explicarse por la existencia de más de un componente activo y/o diferente concentración de los mismos (38). Nosotros estudiamos el efecto antiagregante plaquetario in vitro de extractos acuosos y metanólicos 
de tomate (tipos racimo y "talquino"). Ambos tipos de extractos presentaron actividad antiagregante plaquetaria inducida por ADP, aproximadamente $40 \%$ a $1 \mathrm{mg} / \mathrm{ml}$. Al utilizar colágeno como agonista la inhibición fue menor, y al usar ácido araquidónico y péptido activador del receptor de trombina (TRAP, Thrombin receptor activating peptide) no se observó efecto inhibitorio (39).

Protección del endotelio. En condiciones fisiológicas, el endotelio a través de varias moléculas bioactivas regula la contracción vascular, la adhesión de leucocitos, el crecimiento de células musculares lisas y la agregación plaquetaria (40). La disfunción endotelial, caracterizada por una pérdida parcial o completa del balance entre factores vasoconstrictores (endotelina 1 y angiotensina II) y vasodilatadores (óxido nítrico y prostaciclina) y factores protrombóticos (inhibidor del activador del plasminógeno 1) y antitrombóticos (prostaciclina y heparán sulfato), entre otros aspectos, favorece un estado aterotrombótico, específicamente por la expresión de moléculas de adhesión celular y adhesión de plaquetas. Dicha disfunción endotelial se ve favorecida por las especies reactivas del oxígeno (41), moléculas que son neutralizadas por los llamados antioxidantes. Se ha encontrado que extractos acuosos y metanólicos del tomate presentan actividad antioxidante in vitro (16, 42-45). Los carotenoides ( $\beta$-caroteno, licopeno, zeaxantina, luteína y canxantina), vitamina $\mathrm{C}$ y vitamina $\mathrm{E}$ presentes en tomate pueden presentar actividad antioxidante (46-48). En humanos, se ha descrito recientemente que la suplementación con licopeno reduce el daño oxidativo del ADN y otros marcadores de estrés oxidativo (49). Además se ha reportado que el licopeno inhibe la expresión de ICAM-1 en el endotelio (50).

Efecto antioxidante y antiaterogénico. La teoría más ampliamente aceptada para la génesis de la aterosclerosis en el endotelio vascular propone que la oxidación de la lipoproteína de baja densidad (Low Density Lipoprotein, LDL) juega un rol fundamental (24). Partículas de LDL colesterol (LDL-c) oxidada desencadenan una serie de eventos que llevan al desarrollo de la lesión aterosclerótica $(24,51)$. Se cree que los antioxidantes presentes en los nutrientes disminuyen la progresión de la aterosclerosis por su capacidad para inhibir el daño del proceso oxidativo (52).
El licopeno es un antioxidante que ha sido estudiado ampliamente $(26,53)$. Ensayos in vitro han mostrado que el licopeno puede proteger a la LDL nativa de la oxidación y puede disminuir la síntesis de colesterol $(54,55)$.

Diferentes estudios indican que la regulación lipídica del tomate y los productos derivados del mismo puede ser atribuida al licopeno (55). Sin embargo, otros estudios han mostrado que los niveles de triglicéridos, HDL (High Density Lipoprotein) colesterol (HDL-c) y LDL-c fueron modificados por el consumo de tomate fresco y jugo, pero no por consumo de extracto de licopeno. Esto indica que no solo el licopeno contribuye a mejorar la salud, sino que los compuestos fenólicos también juegan un rol importante (56). Se han descrito varios mecanismos que podrían explicar la disminución del LDL-c asociada a dietas ricas en tomate: inhibición de la 3-hidroxi-3-metilglutaril coenzima-A (HMG CoA) reductasa, aumento de la actividad del receptor de LDL-c y resistencia de la oxidación de la LDL-c:

- Inhibición de la HMG CoA reductasa. Estudios in vitro han mostrado que cultivos de macrófagos conteniendo licopeno inhibieron significativamente la síntesis de colesterol. En el mismo estudio, sujetos suplementados con licopeno por un periodo de tres meses presentaron una reducción significativa de la concentración plasmática de colesterol, mecanismo que podría ser mediado por la inhibición de la actividad de la HMG CoA reductasa (55-57). También se ha observado que al incrementar el consumo de tomate la disminución del colesterol es mayor $(57,58)$.

- Aumento de la actividad del receptor de LDL-c. Se ha mostrado que el licopeno incrementa la actividad del receptor de LDL-c (55), pero, por otra parte, también se ha observado una disminución en la actividad del receptor de LDL en macrófagos expuestos a licopeno (59). Este efecto también ha sido descrito en otros antioxidantes como la vitamina $\mathrm{E}$ y probucol (60-62).

Resistencia a la oxidación de la LDL-c. Otro mecanismo que puede contribuir al efecto antiaterogénico del tomate es la capacidad que le otorga a las partículas de LDL-c de resistir la oxidación (26). En estudios in vitro, el licopeno ha mostrado ser un antioxidante más potente que carotenos $\alpha$ y $\beta$, también presentes en el tomate 
(63). Los efectos protectores del licopeno han sido atribuidos a su actividad antioxidante cuando ésta es medida como la capacidad de neutralización del oxígeno singlete $(57,63)$. El licopeno y otros carotenoides pueden suplementar la actividad antioxidante de la vitamina $\mathrm{E}$, dado que éstos, por su naturaleza lipofílica, se concentran en las fracciones séricas de LDL-c y lipoproteínas de muy baja densidad (Very Low Density Lipoprotein, VLDL-c) $(24,64)$.

\section{TOMATE Y PREVENCIÓN DEL CÁNCER}

Estudios epidemiológicos. El cáncer es un proceso patológico que implica el crecimiento descontrolado de un clon celular maligno; la mayor parte de éstos se producen por mutaciones en los genes que regulan la proliferación celular, la apoptosis y/o los mecanismos de reparación del ADN.

Las modificaciones oxidativas del ADN son una de las causas más importantes del daño mutagénico. En este sentido, moléculas con capacidad antioxidante podrían ser importantes en la prevención de los procesos oxidativos que desencadenan en la oncogénesis (65). Estudios epidemiológicos han mostrado que el consumo de frutas y hortalizas reduce el riesgo a desarrollar algunos tipos de cánceres (7). En dicho contexto, investigaciones han demostrado un efecto protector por parte del tomate en la disminución del riesgo de padecer cáncer (66), especialmente de próstata (67).

Mecanismos. Los carotenoides, entre ellos licopeno, protegen a las plantas del daño oxidativo (68); en humanos estos compuestos consumidos en la dieta son fuente de provitamina A (69) y tienen actividad antioxidante.

Actividad antioxidante. Cuando una molécula se oxida transmite esta condición a otras moléculas amplificando el proceso. Los lípidos de las membranas celulares, las proteínas y el ADN pueden ser oxidados por especies reactivas del oxígeno y del nitrógeno. La capacidad antioxidante del licopeno se debe principalmente al abundante número de dobles enlaces que posee y la capacidad que tiene para perder, mediante rotaciones y vibraciones inter e intramoleculares, la energía obtenida de moléculas oxidantes (70). En cultivos de fibroblastos tratados con peroxynitrito, un potente agente oxidante y mutagénico, el licopeno inhibe la oxidación de proteínas y ADN (71). La mayor parte del licopeno intracelular se localiza en el núcleo (72), lo que concuerda con su rol antimutagénico (71, 73, 74).

Activación de la apoptosis. La apoptosis es un proceso fisiológico normal que tiene como objetivo, entre otras funciones, eliminar células defectuosas o en exceso. Puede ser activado por al menos dos vías: extrínseca (asociada a proteína Fas) e intrínseca o mitocondrial (asociada a la liberación de citocromo C).

El licopeno ha demostrado activar la apoptosis de manera dosisdependiente en una línea celular de cáncer de próstata (LNCaP) (75) de una forma dependiente de citocromo C. En este mismo sentido, el consumo de jugo de tomate ha mostrado un efecto protector en individuos con cáncer de próstata (76), pero no en otros tipos de cánceres (77-79)

Disminución de la proliferación celular. En cada una de las fases del ciclo celular (G1, G2, $\mathrm{S}$ y $\mathrm{M}$ ) existen proteínas que regulan, entre otros procesos, el paso de una fase del ciclo a otra; así, a modo de ejemplo, las proteínas ciclinas D1 y Cdk4 participan en el paso de la fase G1 a la fase $S$ (80). Se ha descrito que el licopeno puede bloquear la fase S de la línea tumoral Hep3B (74), efecto que parece estar mediado por disminución de las ciclinas D1 y E y de la kinasa dependiente de ciclina 4 (Cdka) $(77,81)$. También se ha observado que el licopeno disminuye la proliferación de una línea de cáncer pulmonar disminuyendo la ciclina $\mathrm{E}$ y aumentando las proteínas p21 y p27, reguladores del ciclo celular (82). Este efecto se ha descrito para otras líneas celulares derivadas de tumores, tanto murinos (83) como humanos $(79,84)$. Estudios clínicos han respaldado estas observaciones (85).

Disminución de la angiogénesis y metástasis. La invasión de las células malignas a tejidos extratumorales sanos es un problema para el tratamiento del cáncer. El factor de crecimiento derivado de plaquetas (PDGF) es capaz de estimular la mitogénesis y la angiogénesis $(86,87)$. El PDGF facilita los procesos de angiogénesis y metástasis de diferentes linajes malignos. Se ha observado que el licopeno puede inhibir dicho factor y de esa manera frenar la invasión de células metastásicas (88). Por otro lado, las metaloproteinasas (MMP) son muy 
importantes para el proceso de metástasis dado que éstas rompen las uniones de anclaje de las células malignas al tumor favoreciendo las metástasis. Se ha observado que el licopeno disminuye la actividad de las MMP-2 y MMP-9 (89-91). También se ha descrito este efecto antimetastático del licopeno in vivo (92).

\section{FUTURO}

La baja ingesta de frutas y hortalizas motivó a la OMS y a la FAO a lanzar el año 2003 la iniciativa global para incrementar la producción y consumo hortofrutícola. Esta campaña se inscribe en la Estrategia Mundial sobre Régimen Alimentario, Actividad Física y Salud aprobada el año 2004 por la Asamblea Mundial de la Salud (93). Los países desarrollados se han focalizado en las siguientes acciones: (i) Decidido apoyo gubernamental a la producción primaria y secundaria de productos, en base a incentivos a la producción y agregación de valor de las materias primas, (ii) Fuerte campaña de educación y marketing social, con énfasis en grupos escolares de nivel básico y medio, como también

\section{LITERATURA CITADA}

1. POPKIN, B.M. 2002. An overview on the nutrition transition and its health implications: the Bellagio meeting. Public Health Nutr, 5: 93-103.

2. ALBALA, C.; VIO, F.; KAIN, J.; UAUY, R. 2002. Nutrition transition in Chile: determinants and consequences. Public Health Nutr, 5: 123-128.

3. VIO, F.; ALBALA, C.; CROVETTO, M. 2000. Promoción de salud en la transición epidemiológica de Chile. Revista Chilena de Nutrición, 27: 21-29.

4. ALBALA, C.; VIO, F.; KAIN J.; UAUY, R. 2001. Nutrition transition in Latin America: the case of Chile. Nutr Rev, 59: 170-176.

5. 2003. MINISTERIO DE SALUD DE CHILE. ENCUESTA NACIONAL DE SALUD 2003. Departamento de Salud Pública de la Pontificia Universidad Católica de Chile. Informe Técnico.

6. PALOMO, I.; ICAZA G., MUJICA V., ET AL. 2007. Prevalence of cardiovascular risk factors in adult from Talca, Chile. Rev Med Chil, 135: 904-912.

7. WORLD HEALTH ORGANIZATION W. 2003. Diet nutrition and the prevention of chronic disease.

8. WCRF/AICR. 2007. Food, nutrition, physical activity and the prevention of cancer: a global perspective. United Kingdom, WCRF/AICR.

9. VIO, F.; ZACARÍAS, I.; GONZÁLEZ, D. 2008. Implementación de un programa de promoción del consumo de frutas y verduras: corporación 5 al día Chile. en el segmento poblacional con mayor riesgo de ENT, (iii) Desarrollo de alianzas público-privadas para el mejoramiento de las acciones de promoción de productos en los mercados internacionales y fomento del consumo interno y (iv) Incentivo en investigación, desarrollo e innovación en el sector alimentario para el fomento de nuevas tecnologías en la búsqueda de alimentos de segunda generación para la salud.

El nuevo paradigma del desarrollo agrícola chileno es conseguir que el país sea una potencia alimentaria de nivel mundial. Las nuevas tendencias que orientan la decisión de compra de los consumidores se asocian, mayoritariamente, a la búsqueda de calidad de vida saludable mediante la ingesta de alimentos de alto valor nutritivo, lo que implica una exigencia para la industria alimentaria en el desarrollo de nuevos alimentos funcionales (94). En este contexto, la industria agroindustrial asociada a tomate, tanto nacional como regional, no puede estar ajena a esta tendencia, deberá preocuparse de la presencia del tomate en alimentos funcionales y nutraceúticos, y de apoyar la investigación asociada al estudio de los efectos favorables en la salud humana.
En Contribución de la política agraria al consumo de frutas y verduras en Chile: Un compromiso con la nutrición y la salud de la población. Olivares, S., Leporati, M., Villalobos, P., Barría, L. Editores: 25-42.

10. NUEZ, F. 1995. El cultivo del tomate. Ediciones MundiPrensa, Madrid: 793.

11. RICK, C.M. 1971. The Tomato Ge Locus: Linkage Relations and Geographic Distribution of Alleles. Genetics, 67: 75-85.

12. ORGANIZACIÓN PARA LA AGRICULTURA Y LA ALIMENTACIÓN DE LAS NACIONES UNIDAS, FAO, 2009. Disponible en: <http://www.fao.org/es/ess/ top/commodity.html?item $=388 \&$ lang $=$ es \&year $=2005>$. Consultado 2 de agosto, 2009.

13. ODEPA. 2008. Oficina de Estudios de Políticas Agrarias. www.odepa.cl.

14. GRUSAK, M.A.; DELLAPENNA, D. 1999. Improving the Nutrient Composition of Plants to Enhance Human Nutrition and Health1. Annu Rev Plant Physiol Plant Mol Biol, 50: 133-161.

15. BANHEGYI, G. 2005. [Lycopene--a natural antioxidant]. Orv Hetil, 146: 1621-1624.

16. LENUCCI, M.S.; CADINU, D.; TAURINO, M.; PIRO, G.; DALESSANDRO, G. 2006. Antioxidant composition in cherry and high-pigment tomato cultivars. J Agric Food Chem, 54: 2606-2613. 
17. CANDILLAS, C.G.A.; BAUTISTA-JUSTO, M.; DEL RÍO-OLAGUE, F.; GARCÍA- DÍAZ, C. 2005. Contenido de licopeno en jugo de tomate por aspersión. Revista de ingeniería mexicana 2: 299-307.

18. AHUJA, K.D.; PITTAWAY, J.K.; BALL, M.J. 2006. Effects of olive oil and tomato lycopene combination on serum lycopene, lipid profile, and lipid oxidation. Nutrition, 22: 259-265.

19. UNLU, N.Z.; BOHN, T.; FRANCIS, D.M.; NAGARAJA, H.N., CLINTON, S.K.; SCHWARTZ, S.J. 2007. Lycopene from heat-induced cis-isomer-rich tomato sauce is more bioavailable than from all-trans-rich tomato sauce in human subjects. Br J Nutr, 98: 140-146.

20. MOUSS, M.; LANDRIER, J.F.; REBOUL, E. ET AL. 2008. Lycopene absorption in human intestinal cells and in mice involves scavenger receptor class B type I but not Niemann-Pick C1-like 1. J Nutr, 138: 1432-1436.

21. JAVANMARDI, J.; KUBOTA, C. 2006. Variation of lycopene, antioxidant activity, total soluble solids and weight loss of tomato during postharvest storage. Postharvest Biology and Technology, 41: 151-155.

22. SAAVEDRA, D.R. 2005. Efecto del medio ambiente en el contenido de licopeno y sólidos solubles del tomate para procesamiento. En: Serie Actas, INIA: 45-52.

23. ANZA, M.; RIGA, P. 2007. Efecto de la variedad y de la época de cultivo en la calidad organoléptica y nutricional del tomate. Actas de Horticultura. Sociedad Española de Ciencias Hortícolas, 48.

24. WILLCOX, J.K.; CATIGNANI, G.L.; LAZARUS, S. 2003. Tomatoes and cardiovascular health. Crit Rev Food Sci Nutr, 43: 1-18.

25. ARAB, L.; STECK, S. 2000. Lycopene and cardiovascular disease. Am J Clin Nutr, 71: 1691S-1695S; discussion 1696S-1697S.

26. RAO, A.V. 2002. Lycopene, tomatoes, and the prevention of coronary heart disease. Exp Biol Med (Maywood), 227: 908-913.

27. PARFITT V.J.; RUBBA P.; BOLTON C.; MAROTTA G.; HARTOG M.; MANCINI, M. 1994. A comparison of antioxidant status and free radical peroxidation of plasma lipoproteins in healthy young persons from Naples and Bristol. Eur Heart J, 15: 871-876.

28. KOHLMEIER, L.; KARK, J.D.; GOMEZ-GRACIA, E. ET AL. 1997. Lycopene and myocardial infarction risk in the EURAMIC Study. Am J Epidemiol, 146: 618-626.

29. SANTOS, M.T.; ARANDA, E.; VALLÉS, J.; PALOMO, I. 2005. Hemostasia Primaria. Editorial Universidad de Talca, Talca.

30. ITALIANO, J. 1008. The Structure and Production of Blood Platelets. In: Platelets in Hematologic and Cardiovascular Disorders- A Clinical Handbook. Gresele, P.; Fuster, V.; López, J.; Page, C.; Vermylen, J. (eds.) Cambridge University Press, New York, pp. 1-20.

31. PANES, O.; MATUS, V.; SÁEZ, C.G.; QUIROGA, T.; PEREIRA, J.; MEZZANO, D. 2007. Human platelets synthesize and express functional tissue factor. Blood, 109: 5242-5250.

32. PALOMO, I.; TORO, C.; ALARCÓN, M. 2008. Rol of the platelets in the pathophysiology of atherosclerosis. Molecular Medicine Reports, 1: 179-184.
33. GOLDSCHMIDT, P.; LOPES, N.; CRAWFORD, L.; BECKER, R. 2007. Atherothrombosis and coronary artery disease. Elsevier.

34. ALTMAN, R.; SCAZZIOTA, A.; ROUVIER, J. 2005. Los mecanismos de trombosis. Editorial Akadia, Buenos Aires.

35. DUTTA-ROY,A.K.; CROSBIE, L.; GORDON, M.J. 2001. Effects of tomato extract on human platelet aggregation in vitro. Platelets, 12: 218-227.

36. O'KENNEDY, N.; CROSBIE, L.; VAN LIESHOUT, M.; BROOM, J.I.; WEBB, D.J.; DUTTAROY, A.K. 2006. Effects of antiplatelet components of tomato extract on platelet function in vitro and ex vivo: a time-course cannulation study in healthy humans. Am J Clin Nutr, 84: 570-579.

37. O'KENNEDY, N.; CROSBIE, L.; WHELAN S. ET AL. 2006. Effects of tomato extract on platelet function: a double-blinded crossover study in healthy humans. Am J Clin Nutr, 84: 561-569.

38. YAMAMOTO, J.; TAKA, T.; YAMADA, K. ET AL. 2003. Tomatoes have natural anti-thrombotic effects. Br J Nutr, 90: 1031-1038.

39. GUZMÁN, L. 2009. Estudio de las actividades antiagregante plaquetaria y fibrinolítica in vitro, de extractos de frutas y hortalizas que se consumen en Talca. Tesis de Magíster en Ciencias Biomédicas, Universidad de Talca.

40. BAUMGARTNER-PARZER, S.M.; WALDHAUSL, W.K. 2001. The endothelium as a metabolic and endocrine organ: its relation with insulin resistance. Exp Clin Endocrinol Diabetes, 109 Suppl 2: S166-179.

41. ESPER, R.J.; NORDABY, R.A.; VILARINO, J.O.; PARAGANO, A.; CACHARRON, J.L.; MACHADO, R.A. 2006. Endothelial dysfunction: a comprehensive appraisal. Cardiovasc Diabetol, 5: 4.

42. PALOMO, I.; GUTIÉRREZ, M.; ASTUDILLO, L.; RIVERA, C.; TORRES, C.; GUZMÁN, L.; MOORECARRASCO, R.; CARRASCO, G.; ALARCÓN, M. 2009. Efecto Antioxidante de Frutas y Hortalizas de la Zona Central de Chile. Rev Chil Nutr, 36: 152-158.

43. BOSE, K.S.; AGRAWAL, B.K. 2007. Effect of lycopene from tomatoes (cooked) on plasma antioxidant enzymes, lipid peroxidation rate and lipid profile in grade-I hypertension. Ann Nutr Metab, 51: 477-481.

44. PERIAGO, M.J.; GARCÍA-AlONSO, J.; JACOB, K. ETAL. 2008. Bioactive compounds, folates and antioxidant properties of tomatoes (Lycopersicum esculentum) during vine ripening. Int J Food Sci Nutr: 1-15.

45. JACOB, K.; PERIAGO, M.J.; BOHM, V.; BERRUEZO, G.R. 2008. Influence of lycopene and vitamin $\mathrm{C}$ from tomato juice on biomarkers of oxidative stress and inflammation. Br J Nutr, 99: 137-146.

46. AGARWAL A.; SHEN H.; AGARWAL, S.; RAO, A.V. 2001. Lycopene content of tomato products: Its stability, bioavailability and in vivo antioxidant properties. J Med Food, 4: 9-15.

47. ZAPATA, L.M.G.; L. DAVIES, C.; SCHVAB, M.C. 2007. Estudio de los componentes antioxidantes y actividad antioxidante de tomates. Ciencia, docencia y tecnología, 35.

48. RAFFO, L.M.G.; FOGLIANO, V.; MAIANI, G.; QUAGLIA, G. 2006. Seasonal variations in antioxidant 
components of cherry tomatoes (Lycopersicon esculentum cv. Naomi F1). Journal of Food Composition and Analysis, 19: 11-19.

49. DEVARAJ, S.; MATHUR, S.; BASU, A. ET AL. 2008. A dose-response study on the effects of purified lycopene supplementation on biomarkers of oxidative stress. J Am Coll Nutr, 27: 267-273.

50. HUNG, C.F.; HUANG, T.F.; CHEN, B.H.; SHIEH, J.M.; WU, P.H.; WU, W.B. 2008. Lycopene inhibits TNF-alpha-induced endothelial ICAM-1 expression and monocyte-endothelial adhesion. Eur J Pharmacol, 586: 275-282.

51. MARTIN, K.R.; WU, D.; MEYDANI, M. 2000. The effect of carotenoids on the expression of cell surface adhesion molecules and binding of monocytes to human aortic endothelial cells. Atherosclerosis, 150: 265-274.

52. HELLER, F.R.; DESCAMPS, O.; HONDEKIJN, J.C. 1998. LDL oxidation: therapeutic perspectives. Atherosclerosis, 137 Suppl: S25-31.

53. UPRITCHARD, J.E.; SUTHERLAND, W.H.; MANN, J.I. 2000. Effect of supplementation with tomato juice, vitamin $\mathrm{E}$, and vitamin $\mathrm{C}$ on $\mathrm{LDL}$ oxidation and products of inflammatory activity in type 2 diabetes. Diabetes Care, 23: 733-738.

54. DUGAS, T.R.; MOREL, D.W.; HARRISON, E.H. 1998. Impact of LDL carotenoid and alpha-tocopherol content on LDL oxidation by endothelial cells in culture. J Lipid Res, 39: 999-1007.

55. FUhrMAN, B.; ELIS, A.; AVIRAM, M. 1997. Hypocholesterolemic effect of lycopene and beta-carotene is related to suppression of cholesterol synthesis and augmentation of LDL receptor activity in macrophages. Biochem Biophys Res Commun, 233: 658-662.

56. SHEN, Y.C.; CHEN, S.L.; WANG, C.K. 2007. Contribution of tomato phenolics to antioxidation and down-regulation of blood lipids. J Agric Food Chem, 55: 6475-6481.

57. SILASTE, M.L.; ALFTHAN, G.; ARO, A.; KESANIEMI, Y.A.; HORKKO, S. 2007. Tomato juice decreases LDL cholesterol levels and increases LDL resistance to oxidation. Br J Nutr, 98: 1251-1258.

58. HSU, Y.M.; LAI, C.H.; CHANG, C.Y.; FAN, C.T.; CHEN, C.T.; WU, C.H. 2008. Characterizing the lipid-lowering effects and antioxidant mechanisms of tomato paste. Biosci Biotechnol Biochem, 72: 677-685.

59. NAPOLITANO, M.; DE PASCALE, C.; WHEELERJONES, C.; BOTHAM, K.M.; BRAVO, E. 2007. Effects of lycopene on the induction of foam cell formation by modified LDL. Am J Physiol Endocrinol Metab, 293: E1820-1827.

60. SHIGE, H.; ISHIKAWA, T.; SUZUKAWA, M. ET AL. 1998. Vitamin E reduces cholesterol esterification and uptake of acetylated low density lipoprotein in macrophages. Lipids, 33: 1169-1175.

61. YAMAMOTO, A.; HARA, H.; TAKAICHI, S.; WAKASUGI, J.; TOMIKAWA, M. 1988. Effect of probucol on macrophages, leading to regression of xanthomas and atheromatous vascular lesions. Am J Cardiol, 62: 31B-36B.

62. TEUPSER, D.; THIERY, J.; SEIDEL, D. 1999. Alphatocopherol down-regulates scavenger receptor activity in macrophages. Atherosclerosis, 144: 109-115.
63. DI MASCIO, P.; KAISER, S.; SIES, H. 1989. Lycopene as the most efficient biological carotenoid singlet oxygen quencher. Arch Biochem Biophys, 274: 532-538.

64. PACKER, L.; WEBER, S.U.; RIMBACH, G. 2001. Molecular aspects of alpha-tocotrienol antioxidant action and cell signalling. J Nutr, 131: 369S-373S.

65. ARgiles, J.M.; CARBO, N.; COSTElli, P.; LOPEZ-SORIANO, F.J. 1998. Prevention of cancer and cardiovascular diseases: a common strategy? Med Res Rev, 18: 139-148.

66. MILLS, P.K.; BEESON, W.L.; PHILLIPS, R.L.; FRASER, G.E. 1989. Cohort study of diet, lifestyle, and prostate cancer in Adventist men. Cancer, 64: 598-604.

67. GIOVANNUCCI, E. 2005. Tomato products, lycopene, and prostate cancer: a review of the epidemiological literature. J Nutr, 135: 2030S-2031S.

68. DEMMIG-ADAMS, B.; GILMORE, A.M.; ADAMS, W.W., 3RD. 1996. Carotenoids 3: in vivo function of carotenoids in higher plants. FASEB J, 10: 403-412.

69. DEMMIG-ADAMS, B.; ADAMS, W.W., 3RD. 2002. Antioxidants in photosynthesis and human nutrition. Science, 298: 2149-2153.

70. KRINSKY, N.I. 1992. Mechanism of action of biological antioxidants. Proc Soc Exp Biol Med, 200: 248-254.

71. MUZANDU, K.; ISHIZUKA, M.; SAKAMOTO, K.Q.; ET AL. 2006. Effect of lycopene and beta-carotene on peroxynitrite-mediated cellular modifications. Toxicol Appl Pharmacol, 215: 330-340.

72. LIU,A.; PAJKOVIC, N.; PANG, Y.ET AL. 2006. Absorption and subcellular localization of lycopene in human prostate cancer cells. Mol Cancer Ther, 5: 2879-2885.

73. MUZANDU, K.; EL BOHI, K.; SHABAN, Z.; ISHIZUKA, M.; KAZUSAKA, A.; FUJITA, S. 2005. Lycopene and beta-carotene ameliorate catechol estrogen-mediated DNA damage. Jpn J Vet Res, 52: 173-184.

74. PARK, Y.O.; HWANG, E.S.; MOON, T.W. 2005. The effect of lycopene on cell growth and oxidative DNA damage of Hep3B human hepatoma cells. Biofactors, 23: 129-139.

75. HANTZ, H.L.; YOUNG, L.F.; MARTIN, K.R. 2005. Physiologically attainable concentrations of lycopene induce mitochondrial apoptosis in $\mathrm{LNCaP}$ human prostate cancer cells. Exp Biol Med (Maywood), 230: 171-179.

76. CHEN, L.; STACEWICZ-SAPUNTZAKIS, M.; DUNCAN, C. ET AL. 2001. Oxidative DNA damage in prostate cancer patients consuming tomato sauce-based entrees as a wholefood intervention. J Natl Cancer Inst, 93: 1872-1879.

77. IVANOV, N.I.; COWELL, S.P.; BROWN, P.; RENNIE, P.S.; GUNS, E.S.; COX, M.E. 2007. Lycopene differentially induces quiescence and apoptosis in androgen-responsive and -independent prostate cancer cell lines. Clin Nutr, 26: 252-263.

78. KANAGARAJ, P.; VIJAYABABU, M.R.; RAVISANKAR, B.; ANBALAgAN, J.; ARULdHAS, M.M.; ARUNAKARAN, J. 2007. Effect of lycopene on insulinlike growth factor-I, IGF binding protein-3 and IGF type-I receptor in prostate cancer cells. J Cancer Res Clin Oncol, 133: 351-359.

79. SALMAN, H.; BERGMAN, M.; DJALDETTI, M.; BESSLER, H. 2007. Lycopene affects proliferation and apoptosis of four malignant cell lines. Biomed Pharmacother, 61: 366-369. 
80. DIEHL, J.A. 2002. Cycling to cancer with cyclin D1. Cancer Biol Ther, 1: 226-231.

81. NAHUM, A.; ZELLER, L.; DANILENKO, M. ET AL. 2006. Lycopene inhibition of IGF-induced cancer cell growth depends on the level of cyclin D1. Eur J Nutr, 45: 275-282.

82. LIAN, F.; SMITH, D.E.; ERNST, H.; RUSSELL, R.M.; WANG, X.D. 2007. Apo-10‘-lycopenoic acid inhibits lung cancer cell growth in vitro, and suppresses lung tumorigenesis in the A/J mouse model in vivo. Carcinogenesis, 28: 15671574.

83. GUNASEKERA, R.S.; SEWGOBIND, K.; DESAI, S. ET AL. 2007. Lycopene and lutein inhibit proliferation in rat prostate carcinoma cells. Nutr Cancer, 58: 171-177.

84. FORNELLI, F.; LEONE,A.; VERDESCA, I.; MINERVINI, F.; ZACHEO, G. 2007. The influence of lycopene on the proliferation of human breast cell line (MCF-7). Toxicol In Vitro, 21: 217-223.

85. BARBER, N.J.; ZHANG, X.; ZHU, G. ET AL. 2006. Lycopene inhibits DNA synthesis in primary prostate epithelial cells in vitro and its administration is associated with a reduced prostate-specific antigen velocity in a phase II clinical study. Prostate Cancer Prostatic Dis, 9: 407-413.

86. HELDIN, C.H.; WESTERMARK, B. 1999. Mechanism of action and in vivo role of platelet-derived growth factor. Physiol Rev, 79: 1283-1316.
87. RUITER, D.; BOGENRIEDER, T.; ELDER, D.; HERLYN, M. 2002. Melanoma-stroma interactions: structural and functional aspects. Lancet Oncol, 3: 35-43.

88. CHIANG, H.S.; WU, W.B.; FANG, J.Y. ET AL. 2007. Lycopene inhibits PDGF-BB-induced signaling and migration in human dermal fibroblasts through interaction with PDGF-BB. Life Sci, 81: 1509-1517.

89. HUANG, C.S.; FAN, Y.E.; LIN, C.Y.; HU, M.L. 2007. Lycopene inhibits matrix metalloproteinase-9 expression and down-regulates the binding activity of nuclear factorkappa B and stimulatory protein-1. J Nutr Biochem, 18: 449-456.

90. HUANG, C.S.; SHIH, M.K.; CHUANG, C.H.; HU, M.L. 2005. Lycopene inhibits cell migration and invasion and upregulates $\mathrm{Nm} 23-\mathrm{H} 1$ in a highly invasive hepatocarcinoma, SK-Hep-1 cells. J Nutr, 135: 2119-2123.

91. HWANG, E.S.; LEE, H.J. 2006. Inhibitory effects of lycopene on the adhesion, invasion, and migration of SKHep1 human hepatoma cells. Exp Biol Med (Maywood), 231: 322-327.

92. HUANG, C.S.; LIAO, J.W.; HU, M.L. 2008. Lycopene inhibits experimental metastasis of human hepatoma SKHep-1 cells in athymic nude mice. J Nutr, 138: 538-543.

93. WHO OMDLS. 2004. Estrategia mundial sobre régimen alimentario, actividad física y salud. Ginebra.

94. ROJAS, A.; VILLALOBOS, P.; LEPORATI M. 2008. Desafíos de la Política Alimentaria en la Promoción del Consumo de Frutas y Verduras, Santiago de Chile. 

ANDROS IMPRESORES

www.androsimpresores.cl 
